\title{
Arthroscopic Excision of Heterotopic Ossification in the Supraspinatus Muscle
}

\author{
Lamees A. Altamimi ${ }^{1}$, Erica Kholinne, ${ }^{2,3}$, Hyojune Kim ${ }^{3}$, Dongjun Park ${ }^{3}$, In-Ho Jeon ${ }^{3}$ \\ ${ }^{1}$ King Saud University College of Medicine, Riyadh, Saudi Arabia \\ ${ }^{2}$ Department of Orthopedic Surgery, St. Carolus Hospital, Jakarta, Indonesia \\ ${ }^{3}$ Department of Orthopedic Surgery, Asan Medical Center, University of Ulsan College of Medicine, Seoul, Korea
}

\begin{abstract}
Heterotopic ossification is formation of bone in atypical extra-skeletal tissues and usually occurs spontaneously or following neurologic injury with unknown cause. We report a 46-year-old female with right shoulder pain and restricted range of motion (ROM) for 3 months without history of trauma. Magnetic resonance imaging (MRI) showed a lesion within the rotator cuff supraglenoid. Excisional biopsy from a previous institution revealed a heterotopic ossificans (HO). Following repeat MRI and bone scan, histopathology from arthroscopic resection confirmed an HO. The patient demonstrated improved pain and ROM at follow-up. Idiopathic HO rarely occurs in the shoulder joint, and resection of $\mathrm{HO}$ should be delayed until maturation of the lesion to avoid recurrence. The current case showed that arthroscopic HO resection provides an excellent surgical view to ensure complete lesion removal and minimize soft tissue damage at the supraglenoid area. Furthermore, the minimally invasive procedure of arthroscopy may reduce rehabilitation time and facilitate early return to work.
\end{abstract}

Keywords: Supraspinatus; Heterotopic ossification; Arthroscopy

Heterotopic ossification (HO) is formation of bone in atypical extra-skeletal tissues and usually occurs spontaneously or following neurologic injury resulting from insult to the spinal cord, closed head injury, and burns [1]. Little has been reported regarding idiopathic HO of the shoulder joint. We report a 46-year-old woman with idiopathic $\mathrm{HO}$ in the supraspinatus muscle treated with an arthroscopic excision procedure.

\section{CASE REPORT}

This study was approved by the Institutional Review Board of Asan Medical Center (IRB No. 2019-0736).
A 46-year-old female presented with right shoulder pain that was aggravated by activity and relieved by oral analgesics and who had no history of trauma for the previous 6 months. Three months previously, she had been treated for the same complaint at another hospital, where arthroscopic biopsy revealed HO.

Physical examination revealed a healed scar from previous arthroscopic biopsy. Preoperative range of motion (ROM) for forward elevation was $110^{\circ}$ (active) and $150^{\circ}$ (passive). Shoulder abduction was $110^{\circ}$ (active) and $150^{\circ}$ (passive). External rotation at $90^{\circ}$ abduction was not affected, while internal rotation at $90^{\circ}$ abduction was decreased to $50^{\circ}$ for active motion. Skin color and temperature were unremarkable. Muscle strength grade was 5 of 5

Received: January 18, 2020 Accepted: February 11, 2020

Correspondence to: In-Ho Jeon

Department of Orthopedic Surgery, Asan Medical Center, University of Ulsan College of Medicine, 88 Olympic-ro 43-gil, Songpa-gu, Seoul 05505, Korea Tel: +82-2-3010-3896, Fax: +82-2-488-7877, E-mail: jeonchoi@gmail.com, ORCID: https://orcid.org/0000-0002-9289-9193

Financial support: This research was supported by the Convergence Technology Development Program for Bionic Arm through the National Research Foundation of Korea (NRF) funded by the Ministry of Science \& ICT (No. 2014M3C1B2048422).

Conflict of interest: None. 
for the supraspinatus in external rotation and the subscapularis. The Hawkins impingement test was positive. Laboratory examination demonstrated elevated serum alkaline phosphatase (299 IU/L), elevated C-reactive protein $(7.1 \mathrm{mg} / \mathrm{dL})$, and elevated erythrocyte sedimentation rate $(36 \mathrm{~mm} / \mathrm{hr})$.

Plain shoulder radiographs showed a radiopaque lesion in the subacromial space (Fig. 1A), and magnetic resonance imaging (MRI) showed a soft tissue mass at the subacromial space with an intact rotator cuff tendon. MRI was repeated, and computed tomography (CT) scan and bone scan were obtained. CT scan with three-dimensional reconstruction showed an irregularly-shaped ossification lesion at the dorsal aspect of the coracoid process (Fig. 1B), extending from the supraglenoid tubercle to the coracoid base. Compared with that on the initial plain radiograph, the size of the ossification lesion was increased. The T2-weighted MRI images in coronal oblique and sagittal projections showed a heterogenous soft tissue mass at the rotator interval occupying the subacromial space with supraspinatus tendinosis (Fig. 1C). The rotator cuff tendon was intact, with tendinosis at the supraspinatus. Bone scintigraphy using Technetium-99m (Tcm-methylene diphosphonate) showed a hot spot at the right shoulder, with greater density at the anterior site (Fig. 1D).

For arthroscopic excision, the patient was placed in the beachchair position under general anesthesia with addition of an interscalene block to reduce postoperative pain. The glenohumeral joint was located within the normal limit following a standard diagnostic arthroscopic round. Rotator interval release was performed from the glenohumeral joint to provide a safety margin for release of the ossified lesion due to the proximity to the supraspinatus tendon. Afterward, a standard direct lateral portal was estab-
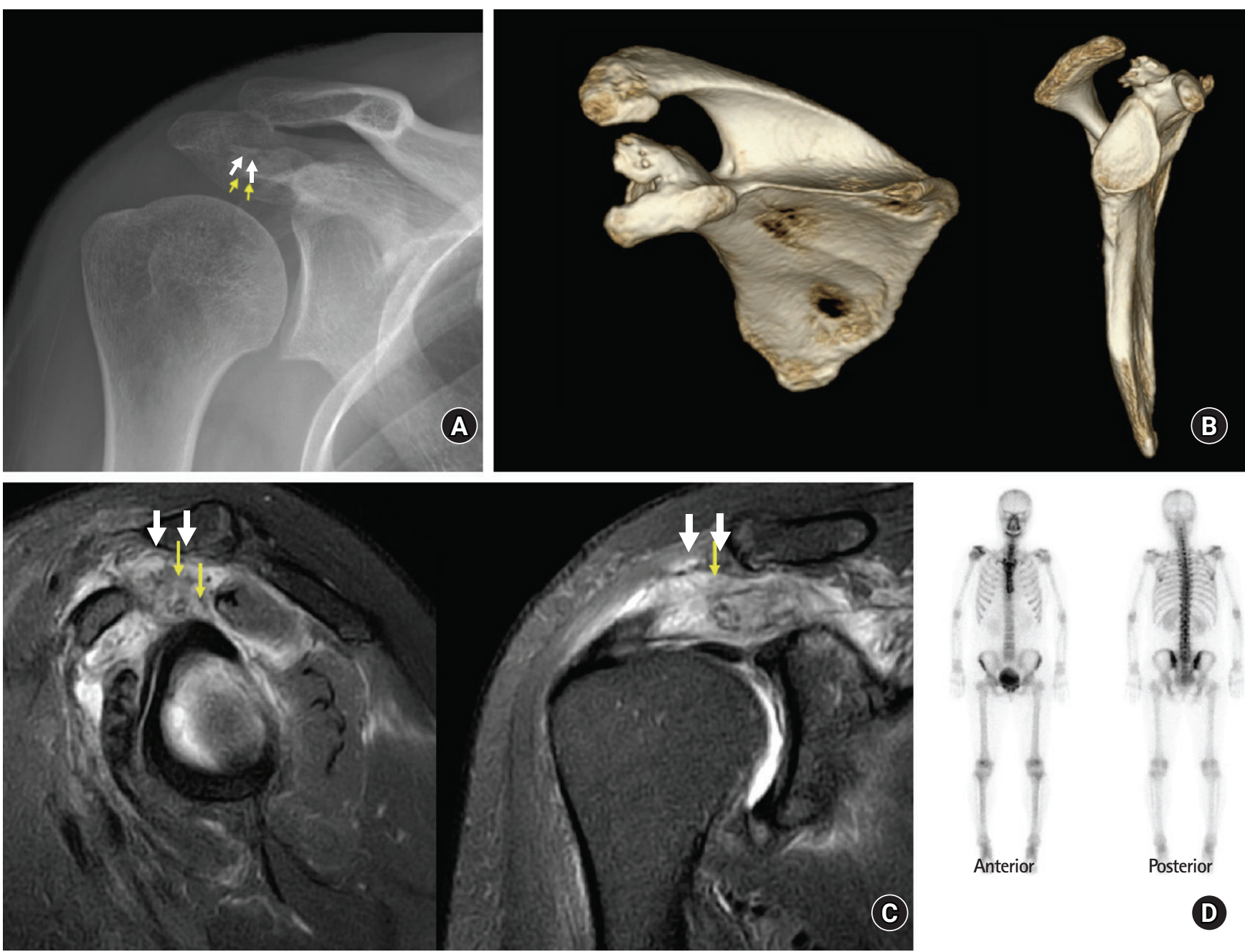

Fig. 1. (A) Diagnostic imaging shows a radio-opaque lesion (arrows) at the subacromial space on plain X-ray. (B) An irregularly-shaped ossification lesion at the dorsal aspect of the coracoid process measuring $7 \mathrm{~mm}$ (mediolateral) $\times 5 \mathrm{~mm}$ (proximodistal) on three-dimensional computed tomography scan. (C) A heterogeneous soft tissue mass (arrows) at the rotator interval occupying the subacromial space on T2-weighted magnetic resonance imaging scan. (D) Hot uptake with greater density at the anterior site on bone-scan. 
lished under the direct vision technique and served as the main viewing portal. Standard anterolateral subacromial decompression was performed following placement of the lateral portal. Ossified tissue with a stalk was connected to the coracoid base (Fig. 2A). Soft tissue release was meticulously performed with radiofrequency ablator and arthroscopic shaver while taking care not to damage the rotator cuff tendon and muscle (Fig. 2B). Resection of the calcified tissue was performed with the defragmentation technique and an arthroscopic burr. The subscapularis muscle remained intact at the end of procedure (Fig. 2C). The resected $\mathrm{HO}$ was sent to pathology, and the final report confirmed heterotopic ossification (Fig. 3).

The patient was discharged on postoperative day two with an arm sling and instructions to exercise as tolerated to increase ROM. A regular follow-up visit 2 weeks after surgery revealed complete pain relief with full ROM. The patient received celecoxib $100 \mathrm{mg}$ (two times daily) for 1 month postoperatively to prevent recurrence of HO. Follow-up plain shoulder radiograph showed absence of the previous lesion (Fig. 4A), and a follow-up CT scan performed 2 months later also showed absence of an ossified lesion in the rotator interval and coracoid base (Fig. 4B).

\section{DISCUSSION}

The literature describes $\mathrm{HO}$ in the shoulder joint associated with events such as head injury, spinal cord injury, severe burns, and postoperative events [2]. However, a report of $\mathrm{HO}$ in the shoulder joint without direct trauma is rare. In the current case, the patient's medical history did not reveal any $\mathrm{HO}$ risk factors, suggesting an idiopathic type of HO. In a case series of 892 patients treated with acromioplasty and distal clavicle resection, Berg and Ciullo [2] reported $5 \%$ with ectopic bone formation, including sites like the subacromial space, acromioclavicular joint, coracoacromial ligament, and coracoclavicular ligament, and approximately $3.2 \%$ of
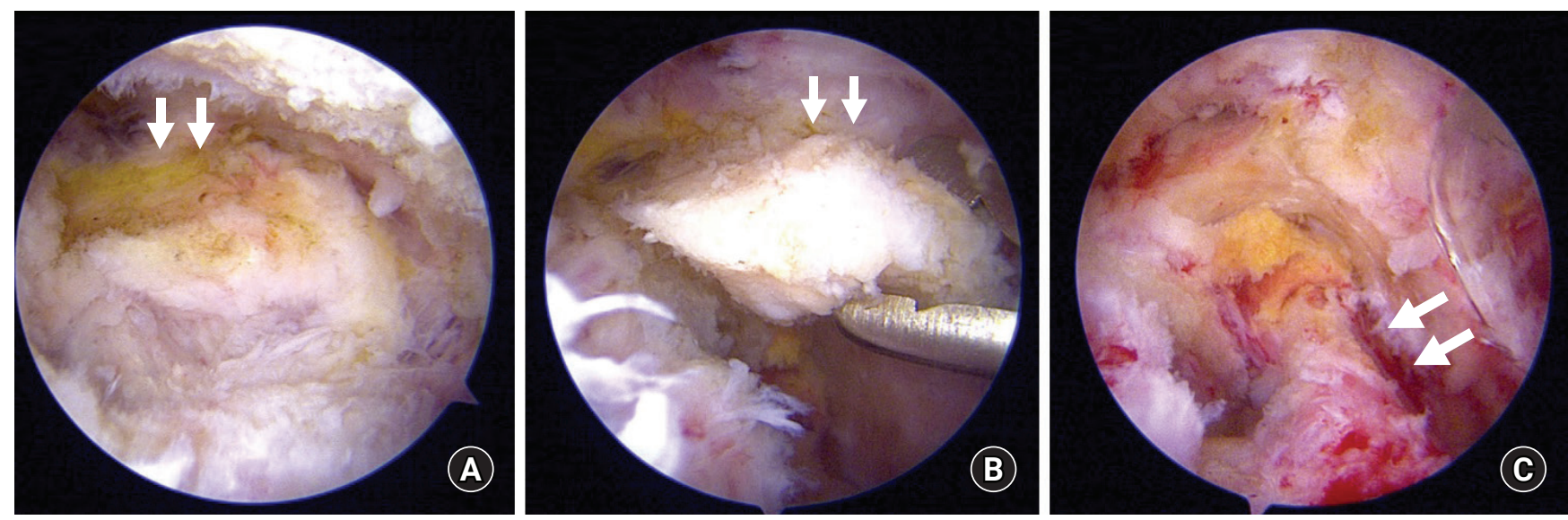

Fig. 2. Arthroscopic view of the right shoulder from the direct lateral portal shows the ossification lesion (arrows) at the acromion undersurface (A), for which soft tissue release was performed for isolation (B). (C) The subscapularis (arrows) was intact following HO excision.

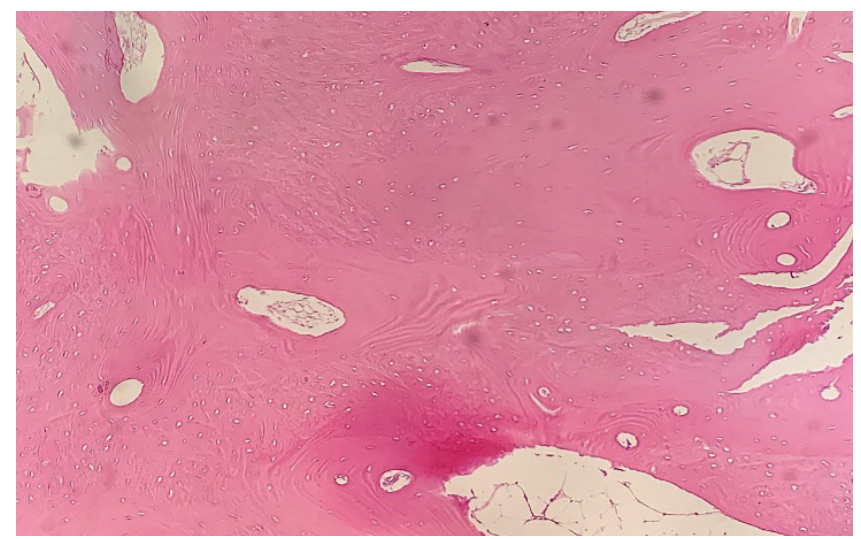

Fig. 3. Histological examination with $H \& E$ staining shows mature trabecular bone $(\times 40)$.
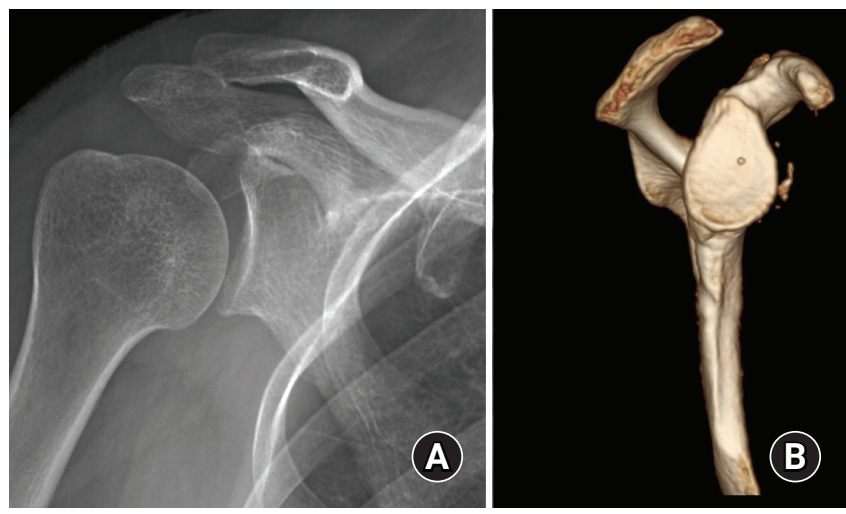

Fig. 4. Follow-up imaging shows no recurrence with absence of the ossification lesion at the previous location on both plain shoulder radiograph (A) and computed tomography scan (B). 
them were symptomatic [2].

$\mathrm{HO}$ is usually asymptomatic [2]. When it is extensive, it may manifest by decreased ROM, localized pain and inflammation of the involved joint, and even bony ankylosis. The process of $\mathrm{HO}$ formation begins within days to weeks after the inciting event. However, it can only be seen on a radiograph 4 weeks after onset. Three-phase bone scintigraphy is the most sensitive imaging modality for early detection of $\mathrm{HO}$, and lesions can be seen as early as 2.5 weeks after injury. Activity on delayed bone scans usually peaks a few months after injury and progressively decreases to normal in a 6- to 12-month period; hence, the classic suggestion is to wait at least 1 year before bone resection [3,4]. Considering the timing for surgical intervention, the definition of complete bony maturation remains inconclusive [3]. Although timing is an important consideration, surgical intervention should be considered when there is lack of functional improvement despite conservative treatment $[3,5]$. One study of the hip joint recommended early surgical excision, as preservation of the tissue planes may help in differentiating ectopic ossifications from normal bone at the site of recent trauma or intervention [6]. Hence, in the present case, early surgical removal of $\mathrm{HO}$ was performed due to significant functional deterioration of the shoulder joint and anticipation of clear margin $\mathrm{HO}$ resection with intact tissue planes. CT and MRI can provide highly detailed anatomic representations of late-stage $\mathrm{HO}$, but they cannot detect the early stages [7].

Posttraumatic HO typically is located inferomedial to the joint [8]. In contrast, the present case demonstrated idiopathic $\mathrm{HO}$ in the supraglenoid area. Classically, post-operative HO has been classified by anatomic location [2], including lesions located in the acromion or coracoacromial ligament (A lesion), such as demonstrated in the current case, and around the clavicle or coracoclavicular ligament (C lesion). Furthermore, additional subtypes were created for lesions that occur in the supraspinatus outlet ("o") or in the acromioclavicular interval (“i”). The HO lesion in the current case was classified as A-o type due to its location at the coracoacromial ligament and occupying the outlet space, which we think caused extrinsic impingement of the supraspinatus outlet.

Recurrence is a known complication following surgical excision of a calcific deposit of the shoulder, with an incidence between $16 \%$ and $18 \%$ [9]. Most recurrence results from incomplete bone removal from a periosteal remnant [2]. Therefore, arthroscopic removal is beneficial for providing an excellent view during the resection procedure, which may lower the recurrence risk. Previous studies recommend a timetable for surgical intervention only for post-traumatic HO [1]. Timing of surgical intervention is key to successful HO surgical treatment and based on maturation of the lesion prior to surgical intervention.

Idiopathic $\mathrm{HO}$ is rare around the shoulder. CT and MRI may provide anatomic location and surgical margins, and bone-scan may confirm maturation state. Arthroscopic HO removal may provide an excellent surgical view to ensure complete surgical resection and minimize soft tissue damage, enabling early return to work.

\section{ORCID}

Lamees A. Altamimi

Erica Kholinne

Hyojune Kim

Dongjun Park

In-Ho Jeon https://orcid.org/0000-0002-9718-2554 https://orcid.org/0000-0001-7665-536X https://orcid.org/0000-0002-4326-8205 https://orcid.org/0000-0003-2331-7097 https://orcid.org/0000-0002-9289-9193

\section{REFERENCES}

1. Pape HC, Marsh S, Morley JR, Krettek C, Giannoudis PV. Current concepts in the development of heterotopic ossification. J Bone Joint Surg Br 2004;86:783-7.

2. Berg EE, Ciullo JV. Heterotopic ossification after acromioplasty and distal clavicle resection. J Shoulder Elbow Surg 1995;4:188-93.

3. Ranganathan K, Loder S, Agarwal S, et al. Heterotopic Ossification: basic-science principles and clinical correlates. J Bone Joint Surg Am 2015;97:1101-11.

4. Garland DE. A clinical perspective on common forms of acquired heterotopic ossification. Clin Orthop Relat Res 1991;(263):13-29.

5. Lee EK, Namdari S, Hosalkar HS, Keenan MA, Baldwin KD. Clinical results of the excision of heterotopic bone around the elbow: a systematic review. J Shoulder Elbow Surg 2013;22:716-22.

6. Genet F, Marmorat JL, Lautridou C, Schnitzler A, Mailhan L, Denormandie P. Impact of late surgical intervention on heterotopic ossification of the hip after traumatic neurological injury. J Bone Joint Surg Br 2009;91:1493-8.

7. Porcellini G, Paladini P, Campi F, Paganelli M. Arthroscopic treatment of calcifying tendinitis of the shoulder: clinical and ultrasonographic follow-up findings at two to five years. J Shoulder Elbow Surg 2004;13:503-8.

8. Boehm TD, Wallace WA, Neumann L. Heterotopic ossification after primary shoulder arthroplasty. J Shoulder Elbow Surg 2005;14:6-10.

9. Wittenberg RH, Rubenthaler F, Wölk T, Ludwig J, Willburger RE, Steffen R. Surgical or conservative treatment for chronic rotator cuff calcifying tendinitis: a matched-pair analysis of 100 patients. Arch Orthop Trauma Surg 2001;121:56-9. 